\title{
Rapid Response Team activation after major hip surgery: patient characteristics and outcomes
}

\section{Angus Pritchard}

Austin Health

Daryl Jones

Austin Health

Rinaldo Bellomo

Austin Health

Andrew Hardidge

Austin Health

lan Harley

Austin Health

Chong Oon Tan

Austin Health

Justin Nazareth

Austin Health

Ranj Guha

Austin Health

Louise Ellard

Austin Health

Raymond Hu

Austin Health

\section{Leonid Churilov}

Florey Institute of Neuroscience and Mental Health - Austin Campus

Laurence Weinberg ( $\Delta$ Laurence.Weinberg@austin.org.au )

Austin Hospital https://orcid.org/0000-0001-7403-7680

Research article

Keywords: rapid response team, medical emergency team, orthopedics, hip arthroplasty, anesthesia, perioperative.

Posted Date: November 3rd, 2020

DOl: https://doi.org/10.21203/rs.3.rs-52563/v2 
License: (c) (i) This work is licensed under a Creative Commons Attribution 4.0 International License. Read Full License 


\section{Abstract}

Background: Rapid response teams (RRTs) are a critical care resource that review deteriorating patients within the hospital. We aimed to describe demographic, preoperative, surgical, anesthetic, and postoperative characteristics of patients who required RRT activation after major hip surgery. We also sought to assess whether these characteristics where associated with mortality during the index hospital admission.

Methods: We reviewed an RRT database of adult patients undergoing orthopedic surgery at a university teaching hospital. We then retrospectively reviewed the medical records to extract a priori-defined patient, preoperative, surgical, anesthetic, and postoperative data of major hip surgery admissions between September 2014 and December 2017. Patients who survived the index hospital stay were compared to those who died.

Results: Overall, 187 patients had postoperative RRT activations. Median (interquartile range) age was 84.0 (78-90) years; 125 (67\%) were female, and most patients had at least one significant comorbidity, median Charlson Comorbidity Index (CCl) 5.0 (4.0-7.0). The majority of patients were frail (68\%), American Society of Anesthesiologists physical status Class 3 or greater (91\%), and underwent nonelective surgery (88\%). Median (interquartile range) time from surgery to RRT activation was 29.4 (11.3-75.0) hours, and 25 (13\%) patients had unplanned admissions to intensive care or high dependency units. Compared to patients who survived RRT activation, those who died displayed higher mean CCl (6.5 [1.8] vs. 5.5 [2.1], $p=0.02)$, were more frail $(80.1 \%$ vs. $56.5 \%, O R=3.2,95 \% \mathrm{Cl}: 1.2,8.1$; $p=0.03$ ), and received less intraoperative opioids (intravenous morphine equi-analgesia: median $=5.8$ $(0.1-8.20$ vs. $11.7(3.7-19.0) \mathrm{mg}, p=0.03)$. They were also more likely to have received an urgent medical review prior to RRT activation ( $62 \%$ vs. $40 \%, O R=2.4,95 \% \mathrm{Cl}: 1.1,5.6) ; p=0.05$.

Conclusions: Death after RRT activation occurred in 1 in 7 patients undergoing major hip surgery. Common patient characteristics included advanced age ( $>82$ years), frailty, high $\mathrm{CCl}$, and emergency surgery. Further studies investigating perioperative surveillance teams in the identification of the high-risk patients before surgery and deteriorating patients after major hip surgery are warranted.

\section{Background}

A rapid response team (RRT) is an interdisciplinary team of critical care health professionals who manage deteriorating patients within the hospital (1). RRTs, also referred to as "medical emergency teams" or "emergency response teams," are commonplace in many modern hospitals. The composition of an RRT can include critical care physicians, anesthetists, critical care nurses, and respiratory therapists (2). Major surgery poses a significant physiological challenge to patients, which in turn can predispose them to an increased risk of postoperative deterioration $(3,4)$. Past case-control and cross-sectional studies have implicated an array of preoperative and anesthetic factors that may be associated with postoperative patient deterioration and the need for RRT activation (5-7). However, these studies provide 
insufficient information on patient characteristics, and preoperative and postoperative anesthesia related variables, including perioperative hemodynamic data, use of fluid, vasoactive drugs, and opioid medications. Furthermore, no studies to date have specifically explored the perioperative factors and patient characteristics of major hip surgery in relation to RRT activation.

Therefore, we sought to describe the perioperative course of patients who underwent major hip surgery and required a postoperative RRT review. We describe patient characteristics and the detailed preoperative, surgical, anesthetic, and postoperative factors of patients who required RRT activation after major hip surgery in an Australian university hospital. Specifically, we evaluated the effects of fluids, vasoactive medications, and opioids on the development of RRT activation and assessed whether these perioperative characteristics affected mortality during the index hospital admission. In addition, we assessed the incidence and severity of perioperative hypotension and whether this was associated with in-hospital mortality. This analysis may facilitate the identification of patients at risk of postoperative deterioration, guide intraoperative patient management, and allow for a focused allocation of critical care and hospital resources, all of which may provide opportunities for proactive prevention strategies.

\section{Methods}

The study was conducted at Austin Health, a tertiary teaching hospital affiliated with the University of Melbourne in Melbourne, Victoria, Australia. Austin Health performs approximately 38,000 surgical procedures annually, including complex cardiothoracic surgery, hepatobiliary-pancreatic surgery, liver transplantation, and major spinal and orthopedic surgery. The orthopedic surgical unit provides services to over 15,000 outpatients annually and performs over 2,500 operations per year, of which approximately 650 are major hip operations.

Following approval from the Austin Health Human Research Ethics Committee (LNR/17/Austin/616), we performed a retrospective cohort study of patients who required RRT activation following major hip surgery between September 2014 and November 2017. The need for informed written consent from participants was waived due to the observational and retrospective nature of the study.

The RRT at our institution is an intensive care-led service introduced in 2000. The RRT is governed by the Department of Intensive Care Medicine, and the RRT team comprises an intensive care registrar and critical care nurse. The RRT is also accompanied by the patient's admitting unit at every activation. Escalation of medical resources to assist with RRT activation are immediately available if required (e.g., anesthesia support for airway management). At our institution, the RRT is activated whenever a patient meets predetermined criteria, which include acute changes in any of the following: obstructed airway, noisy breathing or stridor, problems with a tracheostomy tube, any difficulty in breathing, respiratory rate < 8 or $>25$ breaths/min, oxygen saturation $<90 \%$ despite oxygen administration, heart rate $(<40$ or $>120$ beats/min), systolic blood pressure $<90 \mathrm{mmHg}$, urine output $<50 \mathrm{~mL}$ over 4 hours, sudden change in conscious state, patient cannot be roused, or if any member of staff is worried about imminent deterioration of the patient. Additionally, an RRT is activated for any "code blue." A "code blue" is 
activated whenever a patient suffers a cardiac or respiratory arrest. Our institution's RRT reviews approximately 3,000 patients annually, of which the majority are post-surgery.

For inclusion in our study, patients had to be adults (age > 18 years) undergoing major hip surgery who had an RRT or "code blue" activation post-surgery and were within the index hospital admission. In the case of multiple RRT activations, we only analyzed the first event. We used the following procedures as listed in the International Statistical Classification of Diseases (10th revision) to select patients: total hip arthroplasty, partial hip replacement/hemiarthroplasty (unipolar or bipolar femoral head), revision of hip replacement not otherwise specified, arthrotomy for removal of prosthesis, revision of hip replacement (both acetabular and femoral components), revision of hip replacements (acetabular liner), resurfacing hip (total acetabulum and femoral head), resurfacing hip (partial femoral head or acetabulum), and insertion or removal of any internal fixation device. We excluded superficial procedures of the hip joint including joint arthrocentesis and wound debridement.

As part of routine perioperative care for major hip surgery, patients were assessed by a multidisciplinary team consisting of a surgeon, anesthetist, perioperative physician, and ortho-geriatrician (if over 70 years of age). Routine preoperative investigations included biochemical, hematological, and coagulation tests, and where necessary, all patients were optimized from a cardiorespiratory perspective prior to surgery. All patients underwent preoperative hemoglobin optimization, based on the National Blood Authority of Australia's patient blood management initiative (8). When appropriate, standard perioperative care included strict transfusion practice in accordance with these guidelines. Further, as part of the Diabetes Discovery Initiative, all patients with a $\mathrm{HbA} 1 \mathrm{c}$ of $8.3 \%(67 \mathrm{mmol} / \mathrm{mol})$ and above were seen by the endocrinology unit, which generated a personalized plan for glycemic control according to our institution's guidelines. Patients with a HbA1c between 7.5\% (58 mmol/mol) and 8.2\% (66 mmol/ $\mathrm{mol})$, and those with newly diagnosed diabetes, were seen by a general physician. All patients were managed according to the hospital's perioperative guidelines for patients with diabetes, with an inpatient blood glucose target of 5-10 mmol/L based on the Australian Diabetes Society guidelines (9). In addition, for patients with decision-making capacity, an advance care plan was undertaken, which allowed patients to communicate their future preferences relating to medical treatment to their families, friends, and health professionals. In accordance with existing legislation, a legally defined "person responsible" was appointed to make medical decisions on behalf of a patient who lacks the capacity to give their own consent to treatment.

Data were extracted from the patient's electronic medical records and from Austin Hospital's computerized laboratory results by two independent study investigators. Austin Health uses Cerner electronic medical records, which allows comprehensive electronic data capture and access to patient health information from the perioperative setting. We collected a priori-defined data on patient characteristics, comorbidities, and preoperative management. All other comorbidities were extracted from patient medical records. Patient comorbidity was further defined using the Charlson Comorbidity Index (CCl), a validated metric that predicts 1-year patient mortality (10). For the calculation of the $\mathrm{CCl}$, moderate/severe chronic kidney disease was defined as an estimated glomerular filtration rate of less 
than $60 \mathrm{~mL} / \mathrm{min}$ (Stage 3 or worse), and chronic liver disease was defined based on the Child-Pugh classification (11). Congestive cardiac failure was defined as "heart failure with preserved ejection fraction" (i.e. diagnosed by combination of clinical acumen combined with either echocardiographic features of diastolic dysfunction, or elevated plasma B-type natriuretic peptide concentrations) and "heart failure with reduced ejection fraction" (i.e. left ventricle ejection fraction $<40 \%$ ), regardless of etiology. We used a modified Canadian Study of Health and Aging Clinical Frailty Scale to determine frailty (12).

Intraoperatively, we recorded the type of procedure, anesthesia (regional and/or general), as well as the use of fluids, and vasoactive and opioid medications. Furthermore, the number of epochs of intraoperative hypotension, and the magnitude of each hypotensive event, were recorded. Similar data were collected from the post-anesthesia care unit (PACU). A hypotensive event was defined as any reduction in systolic, diastolic, or mean arterial pressure by $30 \%$ or more as compared to preoperative values; severe hypotension was defined as a reduction in any of the above-mentioned blood pressures by $50 \%$ or more. The duration of hypotensive episodes was not assessed, and each hypotensive measure was counted as a discrete epoch. Postoperatively, we collected postsurgical discharge destination, indication for RRT activation, time to RRT activation from surgery, as well as length of stay and inhospital mortality.

Due to the exploratory and observational design of this study, our primary objectives were to describe the demographic and perioperative profile of patients who required RRT activation after major hip surgery. We also compared the perioperative characteristics of patients who survived the index hospital admission to those who did not. Specifically, we further explored differences between these two groups with respect to the following a priori variables: i) preoperative comorbidities (including frailty), ii) type of anesthesia (general and/or regional), iii) surgical presentation (selective or emergency), iv) perioperative hypotension, and v) use of opioids and vasoactive drugs.

\section{Statistical analysis}

Continuous variables were tested for normality and normally distributed data were expressed as means and standard deviations (SD) and compared using a Student's $t$ test; non-normally distributed data were expressed as medians and interquartile ranges (IQRs) and compared using the Mann-Whitney Utest. Categorical variables were described as proportions and compared using the chi-square test or the Fisher's exact test. All $p$ values of less than 0.05 were treated as indicative of statistical significance, and no correction for multiplicity of testing was undertaken due to the exploratory nature of the study. We reported this study using the STROBE guidelines for reporting observational studies (13). Analyses were performed using GraphPad Prism (version 7.00 for Mac, GraphPad Software, La Jolla, California, United States).

\section{Results}

Over the 3-year period, a total of 8,094 patients underwent elective and nonelective orthopedic surgery at our institution. A total of 1,825 patients underwent surgery on the hip. We excluded 39 patients who 
underwent minor/superficial hip procedures. Consequently, a total of 1,786 patients underwent major hip surgery, of which 187 (9\%) had a postoperative RRT activation. Of these patients, seven ( $0.4 \%)$ fulfilled criteria for a "code blue" activation. Of the seven "code blue" activations, six patients required cardiopulmonary resuscitation, which was unsuccessful in three patients (i.e., death). Three patients had return of spontaneous circulation but remained in the ward and were palliated. The one patient who did not receive CPR was transferred to a critical care setting for further management. The patient was discharged to a residential home on postoperative Day 11. A consort flow diagram is presented in Figure 1 .

Patients who had an RRT activation had a median age and CCl of 84.0 (78.0-90.0) years and 5.0 (4.07.0), respectively; 125 (67\%) patients were female. The majority of patients $(60 \%)$ were frail, American Society of Anesthesiologists (ASA) physical status Class 3 or greater (91\%) and underwent nonelective surgery (88\%). The preoperative mean hemoglobin and albumin values were $119.5(17.7) \mathrm{g} / \mathrm{L}$ and 31.2 (5.5) $\mathrm{g} / \mathrm{L}$, respectively. Median preoperative creatinine was $85(68-108) \mu \mathrm{mol} / \mathrm{L}$. The median time from hospital admission to surgery was $24.7(13.8-38.7)$ hours. The preoperative characteristics of patients undergoing major hip surgery are presented in Table 1.

Table 1. Characteristics and preoperative management of patients undergoing major hip surgery requiring rapid response team activation. 


\section{Total Survived In-hospital \\ $(n=187) \quad(n=161) \quad$ mortality \\ $(n=26)$}

$p$

\section{Demographics}

Sex

Male

Female

Age

Years

Range

BMI $\left(\mathrm{kg} / \mathrm{m}^{2}\right)$

Residence

Home

Low-level care

High-level care

\section{Frailty (modified CSHA CFS)}

Fit, well, or vulnerable

Frail (mild, moderate, or severe)

ASA physical status classification

$$
\begin{aligned}
& <3 \\
& \geq 3
\end{aligned}
$$

1

2

3

4

Presentation

Emergency

Elective
$62(33 \%)$

$125(67 \%) \quad 109(68 \%)$

$52(32 \%)$
$109(68 \%)$

0.65

$10(38 \%)$

$16(62 \%)$

0.17

$84.0(78.0-\quad 84.0(77.0-\quad 85.5(8.77)$

$\begin{array}{ccc}90.0) & 90.0) & \\ 20-100 & 20-100 & 56-99 \\ 24.7(6.2) & 25.0(6.2) & 23.2(6.3) \\ & & \\ 128(68 \%) & 113(70 \%) & 15(58 \%) \\ 39(21 \%) & 34(21 \%) & 5(19 \%) \\ 20(11 \%) & 14(9 \%) & 6(23 \%)\end{array}$

$74(40 \%) \quad 70(43 \%) \quad 5(19 \%)$

$113(60 \%) \quad 91(57 \%) \quad 21(81 \%)$

$\begin{array}{cccc}17(9 \%) & 16(10 \%) & 1(4 \%) & 0.48 \\ 170(91 \%) & 145(90 \%) & 25(96 \%) & \end{array}$

$1(0.5 \%) \quad 1(0.6 \%)$

0

0.13

$\begin{array}{ccc}16(9 \%) & 15(9 \%) & 1(4 \%) \\ 110(59 \%) & 98(61 \%) & 12(46 \%) \\ 60(32 \%) & 47(29 \%) & 13(50 \%)\end{array}$

0.32

Comorbidities 


\begin{tabular}{|c|c|c|c|c|}
\hline & $\begin{array}{c}\text { Total } \\
(n=187)\end{array}$ & $\begin{array}{l}\text { Survived } \\
(n=161)\end{array}$ & $\begin{array}{l}\text { In-hospital } \\
\text { mortality } \\
(n=26)\end{array}$ & $p$ \\
\hline Charlson Comorbidity Index & $\begin{array}{c}5.0(4.0- \\
7.0)\end{array}$ & $\begin{array}{c}5.0(4.0- \\
7.0)\end{array}$ & $6.0(5.0-7.25)$ & 0.01 \\
\hline Diabetes & $31(17 \%)$ & $28(17 \%)$ & $3(12 \%)$ & 0.58 \\
\hline Chronic liver disease & $4(2 \%)$ & $4(3 \%)$ & 0 & 0.64 \\
\hline Moderate/severe liver disease & $2(1 \%)$ & $2(1 \%)$ & 0 & 0.99 \\
\hline $\begin{array}{l}\text { Malignancy (solid tumors, lymphoma, } \\
\text { leukemia) }\end{array}$ & $40(21 \%)$ & $28(17 \%)$ & $12(46 \%)$ & 0.002 \\
\hline Metastatic malignancy & $5(3 \%)$ & $4(3 \%)$ & $1(4 \%)$ & 0.99 \\
\hline Congestive cardiac failure & $34(18 \%)$ & $29(18 \%)$ & $5(19 \%)$ & 0.99 \\
\hline Myocardial infarction & $13(7 \%)$ & $12(8 \%)$ & $1(4 \%)$ & 0.70 \\
\hline Chronic obstructive pulmonary disease & $41(22 \%)$ & $35(22 \%)$ & $6(23 \%)$ & 0.99 \\
\hline $\begin{array}{l}\text { Cerebral vascular accident / transient } \\
\text { ischemic attack }\end{array}$ & $26(14 \%)$ & $22(14 \%)$ & $4(15 \%)$ & 0.99 \\
\hline Dementia & $36(19 \%)$ & $28(17 \%)$ & $8(31 \%)$ & 0.18 \\
\hline Hemiplegia & $1(0.5 \%)$ & $1(0.6 \%)$ & 0 & 0.99 \\
\hline Peripheral vascular disease & $9(5 \%)$ & $7(4 \%)$ & $2(8 \%)$ & 0.62 \\
\hline \multicolumn{5}{|l|}{ Preoperative paradigm } \\
\hline Admission to surgery (hr) & $\begin{array}{c}24.7(13.8- \\
38.7)\end{array}$ & $\begin{array}{l}24.7(13.5- \\
38.2)\end{array}$ & $\begin{array}{c}25.7(18.4- \\
46.5)\end{array}$ & 0.15 \\
\hline Preadmission nerve block & $113(60 \%)$ & $93(58 \%)$ & $20(77 \%)$ & 0.08 \\
\hline Preoperative albumin (g/L) & $31.2(5.5)$ & $31.3(5.7)$ & $31.1(4.5)$ & 0.95 \\
\hline Preoperative hemoglobin (g/L) & $\begin{array}{l}119.5 \\
(17.7)\end{array}$ & $\begin{array}{l}119.7 \\
(16.5)\end{array}$ & $118.6(24.0)$ & 0.85 \\
\hline Preoperative creatinine $(\mu \mathrm{mol} / \mathrm{L})$ & $\begin{array}{c}85(68- \\
108.0)\end{array}$ & $\begin{array}{c}86(67- \\
108.0)\end{array}$ & $82(70-116.8)$ & 0.67 \\
\hline
\end{tabular}

Note. Data are presented as number (proportion), mean (standard deviation), or median (interquartile range). $\mathrm{BMI}=$ body mass index; CSHA CFS = Canadian Study of Health and Aging Clinical Frailty Scale; ASA = American Society of Anesthesiologists. 
The operative course of patients who underwent an RRT activation is summarized in Table 2. Most patients underwent hip arthroplasty, with $40 \%$ of these being total hip arthroplasty. One quarter had surgery out of hours (between $18 \mathrm{~h} 00$ and $08 \mathrm{~h} 00$, or over a weekend). Almost 1 in 3 patients had surgery performed under regional anesthesia, one third under general anesthesia, and the rest had combined general and regional anesthesia. The median duration of surgery was 128 (99-163) minutes. Median intraoperative intravenous morphine equi-analgesia dose was 10.0 (2-16.7) $\mathrm{mg}$. A detailed breakdown of opioid use is presented in Table 2. The mean lowest recorded temperature in theater was $36.2(0.4){ }^{\circ} \mathrm{C}$.

Table 2. Intraoperative variables of patients undergoing major hip surgery requiring rapid response team activation. 


\begin{tabular}{|c|c|c|c|c|}
\hline & $\begin{array}{c}\text { Total } \\
(n=187)\end{array}$ & $\begin{array}{l}\text { Survived } \\
(n=161)\end{array}$ & $\begin{array}{c}\text { In-hospital } \\
\text { mortality } \\
(n=26)\end{array}$ & $p$ \\
\hline Procedure type & & & & 0.63 \\
\hline Total hip replacement & $40(21 \%)$ & $37(23 \%)$ & $3(12 \%)$ & \\
\hline Partial hip replacement & $60(31 \%)$ & $51(31 \%)$ & $9(35 \%)$ & \\
\hline Intramedullary nail & $55(28 \%)$ & $46(28 \%)$ & $9(35 \%)$ & \\
\hline Dynamic hip screw & $38(20 \%)$ & $29(18 \%)$ & $5(19 \%)$ & \\
\hline Surgical approach & & & & 0.90 \\
\hline Anterior & $2(1 \%)$ & $2(1 \%)$ & 0 & \\
\hline Anterolateral & $9(5 \%)$ & $8(5 \%)$ & $1(4 \%)$ & \\
\hline Lateral & $102(55 \%)$ & $86(53 \%)$ & $16(62 \%)$ & \\
\hline Posterolateral & $1(0.5 \%)$ & $1(0.6 \%)$ & 0 & \\
\hline Posterior & $36(19 \%)$ & $32(20 \%)$ & $4(15 \%)$ & \\
\hline \multicolumn{5}{|l|}{ Procedure } \\
\hline Duration (min) & $\begin{array}{c}128(99- \\
163)\end{array}$ & $\begin{array}{c}125(95- \\
158.5)\end{array}$ & $\begin{array}{c}150(117.3- \\
189.3)\end{array}$ & 0.07 \\
\hline After-hours surgery $(18 \mathrm{~h} 00-08 \mathrm{~h} 00)$ & $42(23 \%)$ & $35(22 \%)$ & $6(23 \%)$ & 0.99 \\
\hline Anesthesia & & & & 0.78 \\
\hline General only & $60(32 \%)$ & $53(33 \%)$ & $7(27 \%)$ & \\
\hline Regional only & $55(29 \%)$ & $46(29 \%)$ & $9(35 \%)$ & \\
\hline Combined regional and general & $72(40 \%)$ & $62(39 \%)$ & $10(38 \%)$ & \\
\hline Regional technique ${ }^{a}$ & & & & 0.004 \\
\hline Spinal & $66(52 \%)$ & $56(52 \%)$ & $10(52 \%)$ & \\
\hline Epidural & $2(2 \%)$ & 0 & $2(10 \%)$ & \\
\hline Femoral or fascia iliaca block & $66(52 \%)$ & $57(53 \%)$ & $9(47 \%)$ & \\
\hline Airway management & & & & 0.71 \\
\hline Endotracheal tube & $110(59 \%)$ & $97(60 \%)$ & $13(50 \%)$ & \\
\hline Supraglottic device & $19(10 \%)$ & $16(10 \%)$ & $3(12 \%)$ & \\
\hline
\end{tabular}

\section{Temperature}

Intraoperative lowest $\left({ }^{\circ} \mathrm{C}\right)^{b}$

$36.2(0.4) \quad 36.2(0.4)$

$36.1(0.3)$

0.80 


\begin{tabular}{|c|c|c|c|c|}
\hline & $\begin{array}{c}\text { Total } \\
(n=187)\end{array}$ & $\begin{array}{l}\text { Survived } \\
(n=161)\end{array}$ & $\begin{array}{c}\text { In-hospital } \\
\text { mortality } \\
(n=26)\end{array}$ & $p$ \\
\hline \multicolumn{5}{|l|}{ Opioids } \\
\hline Patients receiving opioids & $146(78 \%)$ & 129 (80\%) & $17(65 \%)$ & 0.12 \\
\hline $\begin{array}{l}\text { Intravenous morphine equi- } \\
\text { analgesia dose (mg) }\end{array}$ & $\begin{array}{c}10.0(2- \\
16.7)\end{array}$ & $\begin{array}{c}11.7(3.7- \\
19)\end{array}$ & $5.8(0.1-8.2)$ & 0.03 \\
\hline \multicolumn{5}{|l|}{ Fentanyl } \\
\hline Patients & $132(71 \%)$ & 116 (72\%) & $16(62 \%)$ & \\
\hline Total dose $(\mu \mathrm{g})$ & $\begin{array}{c}200(102.5- \\
300)\end{array}$ & $\begin{array}{c}200(125- \\
300)\end{array}$ & $125(100-230)$ & \\
\hline \multicolumn{5}{|l|}{ Morphine } \\
\hline Patients & $13(7 \%)$ & $12(8 \%)$ & $1(4 \%)$ & \\
\hline Total dose (mg) & $10(5.75-10)$ & $\begin{array}{c}9(5.125- \\
10)\end{array}$ & $10(10-10)$ & \\
\hline \multicolumn{5}{|l|}{ Oxycodone } \\
\hline Patients & $6(3 \%)$ & $6(4 \%)$ & 0 & \\
\hline Total dose (mg) & $7(3.5-10)$ & $7(3.5-10)$ & & \\
\hline \multicolumn{5}{|l|}{ Alfentanil } \\
\hline Patients & $17(10 \%)$ & $14(9 \%)$ & $3(12 \%)$ & \\
\hline Total dose $(\mu \mathrm{g})$ & $\begin{array}{c}750(350- \\
1000)\end{array}$ & $\begin{array}{c}675(375- \\
1000)\end{array}$ & $\begin{array}{c}1000(250- \\
1000)\end{array}$ & \\
\hline \multicolumn{5}{|l|}{ Other drugs } \\
\hline \multicolumn{5}{|l|}{ Tramadol } \\
\hline Patients & $2(1 \%)$ & $2(1 \%)$ & 0 & \\
\hline Total dose (mg) & $\begin{array}{c}150(100- \\
150)\end{array}$ & $\begin{array}{c}150(100- \\
200)\end{array}$ & & \\
\hline \multicolumn{5}{|l|}{ Clonidine } \\
\hline Patients & $7(4 \%)$ & $7(4 \%)$ & 0 & \\
\hline Total dose (mg) & $45(45-60)$ & $45(45-60)$ & & \\
\hline
\end{tabular}


Note. Data are presented as number (proportion), mean (standard deviation), or median (interquartile range).

a $n=127,108$, and 19 for total, survived, and did not survive, respectively.

${ }^{\mathrm{b}}$ Missing values, $n=30$.

During anesthesia, two thirds of patients had received an arterial line as part of intraoperative advanced hemodynamic monitoring. Almost all patients had received intraoperative vasopressor support, and $58 \%$ had a documented intraoperative hypotensive event. Of those patients who were hypotensive, the median number of hypotensive episodes was 3.5 (1-9). A detailed overview is presented in Table 3.

Table 3. Hemodynamics in patients undergoing major hip surgery requiring rapid response team activation. 


\section{Total \\ Survived \\ In-hospital \\ $(n=187) \quad(n=161) \quad$ mortality \\ $(n=26)$}

$p$

\section{Preinduction blood pressure ( $\mathrm{mmHg}$ )}

Systolic

$\begin{array}{cccc}135(22) & 135(22) & 134(23) & 0.68 \\ 93(15) & 92(13) & 94(15) & 0.49 \\ 72(15) & 71(12) & 75(13) & 0.12\end{array}$

\section{Intraoperative hypotension}

Patients with hypotension

Epochs per patient

of systolic hypotension

of diastolic hypotension

of systolic and diastolic

hypotension

Patients with severe hypotension

$16(9 \%)$

$13(8 \%)$

$3(12 \%)$

0.99

Severe hypotensive epochs per

$1(1-2)$

$1(1-2.5)$

$1(1-2)$

0.67 patient

Continuous arterial blood pressure $127(68 \%)$

$105(65 \%)$

$22(85 \%)$

0.07 monitoring

\section{Intraoperative fluid administration}

Total fluids $(\mathrm{mL})$

$\begin{array}{cccc}1000(1000- & 1000(1000- & 1000(1000- & 0.46 \\ 1900) & 1875) & 1925) & \end{array}$

Crystalloid

Patients receiving

$177(95 \%) \quad 151(94 \%) \quad 26(100 \%) \quad 0.36$

Volume received $(\mathrm{mL})$ $1000(1000-\quad 1000(1000-\quad 1000(1000-\quad 0.36$

1500) 1500)

$4 \%$ albumin

Patients receiving

$\begin{array}{cccc}12(6 \%) & 12(8 \%) & 0 & 0.22 \\ 250(250- & 250(250- & & >0.99\end{array}$

Volume received $(\mathrm{mL})$

500)

500)

Blood transfusion

Patients receiving

$12(6 \%)$

$10(6 \%)$

$2(8 \%)$

0.99 


\begin{tabular}{|c|c|c|c|c|}
\hline & $\begin{array}{c}\text { Total } \\
(n=187)\end{array}$ & $\begin{array}{l}\text { Survived } \\
(n=161)\end{array}$ & $\begin{array}{l}\text { In-hospital } \\
\text { mortality } \\
(n=26)\end{array}$ & $p$ \\
\hline Units received & $1(1-2)$ & $1(1-1.63)$ & $2.5(2-3)$ & 0.08 \\
\hline
\end{tabular}

\section{Intraoperative vasoactive medication}

Total patients receiving any

$164(88 \%)$

$142(88 \%)$

$22(85 \%)$

0.75

vasoactive medication

Metaraminol

Patients receiving

Dose received (mg)

$\begin{array}{cr}149(80 \%) & 128(80 \%) \\ 2.25(1.25- & 2.63(1.5- \\ 4.0) & 4.19)\end{array}$

Ephedrine

Patients receiving

$46(25 \%)$

$40(25 \%)$

$6(23 \%)$

Dose received (mg)

$10(6-21.5) \quad 10(6-22.5)$

11 (5.75-

0.85

21.75)

Phenylephrine

Patients receiving

Dose received (mg)

$1(0.5 \%)$

$1(0.6 \%)$

0

$10(10-10) \quad 10(10-10)$

Noradrenaline

Patients receiving

$1(0.5 \%)$

$1(0.6 \%)$

0

Dose received $(\mu \mathrm{g})$

1405 (1405- 1405 (1405-

$$
\text { 1405) 1405) }
$$

Adrenaline

Patients receiving

$1(0.5 \%)$

$1(0.6 \%)$

0

Dose received $(\mu \mathrm{g})$

$40(40-40)$

$40(40-40)$ $>0.99$

Atropine

Patients receiving

Dose received $(\mu g)$

$\begin{array}{ccc}3(2 \%) & 2(1 \%) & 1(4 \%) \\ 600(300- & 600(600- & 300(300- \\ 600) & 600) & 300)\end{array}$

0.36

\section{Post-anesthesia care unit}

Patients with hypotension

$73(45 \%)$

$61(38 \%)$

$12(46 \%)$

0.50

Epochs per patient

$2(1-4)$

$2(1-4)$

$2.5(1-4.5)$

0.50 


\begin{tabular}{ccccc}
\hline & $\begin{array}{c}\text { Total } \\
(\boldsymbol{n}=\mathbf{1 8 7})\end{array}$ & $\begin{array}{c}\text { Survived } \\
(\boldsymbol{n}=\mathbf{1 6 1 )}\end{array}$ & $\begin{array}{c}\text { In-hospital } \\
\text { mortality } \\
(\boldsymbol{n}=\mathbf{2 6})\end{array}$ & $\boldsymbol{p}$ \\
\hline $\begin{array}{c}\text { of systolic hypotension } \\
\text { of diastolic hypotension }\end{array}$ & $1(1-1.5)$ & $1(1-1)$ & $2(2-2)$ & 0.16 \\
$\begin{array}{c}\text { of systolic and diastolic } \\
\text { hypotension }\end{array}$ & $2(1-4)$ & $2(1-4)$ & $1(1-4)$ & 0.14 \\
Vasopressor use & $2(1-4.5)$ & $2(1-5)$ & $2(1.25-3.75)$ & 0.21 \\
\hline
\end{tabular}

Note. Data are presented as number (proportion), mean (standard deviation), or median (interquartile range).

Postoperatively, in the PACU, $10 \%$ of patients had received vasopressor support, and $45 \%$ of patients had a documented hypotensive episode; the median number of hypotensive epochs was 2 (1-4). An overview of hypotension and vasopressor use in PACU is presented in Table 3. Almost all patients were transferred directly to a standard surgical ward; seven (4\%) patients, directly to critical care services (intensive care unit [ICU] or high dependency unit [HDU]). The median time to RRT activation from discharge from theater was 29.4 (11.3-75) hours. The majority of RRT activations occurred within the first 48 hours of surgery. The most common reason for RRT activation was hypotension (35\%), followed by tachycardia (25\%), and high respiratory rate (11\%). Most of the RRT activations occurred after hours, and 25 (13\%) patients had unplanned admissions to critical care services (ICU or HDU) after RRT activation. Severe pain was not a cause RRT activation in any patient. Of those who survived, the median length of hospital stay was 9 (614) days. Overall, 26 (14\%) patients did not survive their acute hospital admission and died a median of $2.9(0.2-8.9)$ days after RRT review. The cause of death was available from the Coroner's report for 23 patients and from attending clinician's death certificate in 3 patients. Causes of death included: complications following surgery in a patient with comorbidity [n=11], ischemic heart disease [ $n=4]$, cerebral vascular event [ $n=1]$, pulmonary embolus [ $n=1]$, progression of multiple myeloma [ $n=1]$, ischemic heart disease and perforated bowel [ $n=1]$, unascertained/natural [ $n=3]$, non-small cell lung cancer $[n=1]$, ischemic heart disease and urosepsis [ $n=1]$, aortic dissection rupture [ $n=1)]$, exacerbation of chronic obstructive pulmonary disease [ $n=1]$. A detailed overview of RRT activations and patient outcomes is presented in Table 4.

Table 4. Postoperative outcomes in patients undergoing major hip surgery requiring rapid response team activation. 


\section{Total \\ Survived \\ In-hospital \\ $(n=187) \quad(n=161) \quad$ mortality \\ $(n=26)$}

$p$

\section{PACU temperature $\left({ }^{\circ} \mathrm{C}\right)$}

On arrival

$\begin{array}{cccc}36.3(0.4) & 36.3(0.4) & 36.4(0.3) & 0.12 \\ 4(2 \%) & 4(3 \%) & 0 & 0.64\end{array}$

$<35.5$

PACU analgesia

PACU analgesia

Patients receiving opioids

Intravenous morphine equi-analgesia (mg)

Fentanyl

Patients

Total dose $(\mu \mathrm{g})$

Morphine

Patients

Total dose (mg)

Oxycodone

Patients

Total dose (mg)

Tramadol

Patients

Total dose (mg)

Discharge location

Ward

HDU

ICU

\section{RRT call}

Need for urgent medical review prior to RRT activation

$\begin{array}{cccc}71(38 \%) & 62(39 \%) & 9(35 \%) & 0.67 \\ 56(30 \%) & 48(30 \%) & 8(31 \%) & 0.99 \\ 4(2.7- & 4(2.7-6.7) & 3(1.3-4.8) & 0.84 \\ 6.7) & & & \end{array}$

$\begin{array}{ccc}46(25 \%) & 38(24 \%) & 8(31 \%) \\ 50(30- & 50(37.5- & 45(20-72.5) \\ 80) & 80) & \end{array}$

$8(4 \%)$

$8(5 \%)$

0

$8(5-10) \quad 8(5-10)$

$3(2 \%)$

$3(2 \%)$

0

$10(4-50) \quad 10(4-50)$

$\begin{array}{cc}3(2 \%) & 3(2 \%) \\ 200(100- & 200(100- \\ 200) & 200)\end{array}$

0

0.90

$\begin{array}{ccc}180(96 \%) & 155(96 \%) & 25(96 \%) \\ 4(2 \%) & 3(2 \%) & 1(4 \%) \\ 3(2 \%) & 3(2 \%) & 0\end{array}$

$80(43 \%)$

$64(40 \%)$

$16(62 \%)$

0.05 


\begin{tabular}{|c|c|c|c|c|}
\hline & $\begin{array}{c}\text { Total } \\
(n=187)\end{array}$ & $\begin{array}{l}\text { Survived } \\
(n=161)\end{array}$ & $\begin{array}{c}\text { In-hospital } \\
\text { mortality } \\
(n=26)\end{array}$ & $p$ \\
\hline $\begin{array}{l}\text { Time from end of surgery to RRT } \\
\text { activation (hr) }\end{array}$ & $\begin{array}{c}29.4 \\
(11.3-75)\end{array}$ & $\begin{array}{c}29.4(10.8- \\
68.5)\end{array}$ & $\begin{array}{c}29.3(15.8- \\
107.5)\end{array}$ & 0.36 \\
\hline$<12 \mathrm{hr}$ & $42(25 \%)$ & $39(24 \%)$ & $3(12 \%)$ & 0.21 \\
\hline$<24 \mathrm{hr}$ & $81(43 \%)$ & $71(44 \%)$ & $10(43 \%)$ & 0.44 \\
\hline$<48 \mathrm{hr}$ & $122(65 \%)$ & $106(66 \%)$ & $16(62 \%)$ & 0.99 \\
\hline Out-of-hours RRT (18h00-08h00) & $101(54 \%)$ & $90(56 \%)$ & $11(42 \%)$ & 0.21 \\
\hline \multicolumn{5}{|l|}{ Cause for RRT activation } \\
\hline Hypotension & $65(35 \%)$ & $61(38 \%)$ & $4(15 \%)$ & \\
\hline Bradycardia & $3(1.5 \%)$ & 0 & 0 & \\
\hline Tachycardia & $47(25 \%)$ & $42(26 \%)$ & $5(19 \%)$ & \\
\hline Low respiratory rate & $3(2 \%)$ & $3(2 \%)$ & 0 & \\
\hline High respiratory rate & $21(11 \%)$ & $16(10 \%)$ & $5(19 \%)$ & \\
\hline Breathing difficulties & $3(2 \%)$ & $1(0.5 \%)$ & $3(12 \%)$ & \\
\hline Worried (clinical concern) & $15(8 \%)$ & $13(8 \%)$ & $1(4 \%)$ & \\
\hline Low oxygen saturations & $15(8 \%)$ & $13(8 \%)$ & $2(8 \%)$ & \\
\hline Change in conscious state & $10(5 \%)$ & $9(6 \%)$ & $4(15 \%)$ & \\
\hline Severe or uncontrolled pain & $2(1 \%)$ & $1(0.5 \%)$ & $1(4 \%)$ & \\
\hline Low urine output & $3(1.5 \%)$ & $2(1 \%)$ & $1(4 \%)$ & \\
\hline \multicolumn{5}{|l|}{ Remaining hospital stay } \\
\hline Unplanned admission to HDU or ICU & $25(13 \%)$ & $22(14 \%)$ & $3(12 \%)$ & 0.99 \\
\hline Days from surgery to discharge & & $9(6-14)$ & & \\
\hline Time to death after RRT (days) & & & $2.9(0.2-8.9)$ & \\
\hline
\end{tabular}

Note. Data are presented as number (proportion), mean (standard deviation), or median (interquartile range). PACU = post-anesthesia care unit; $\mathrm{RRT}=$ rapid response team; HDU = high dependency unit; ICU = intensive care unit.

Patients who did not survive admission had a higher median $\mathrm{CCl}(6.0$ [5.0-7.3] vs. $5.0[4.0-7.0], p=0.01)$, were more likely to be frail ( $80.1 \%$ vs. $56.5 \%, O R=3.2, p=0.03,95 \% \mathrm{Cl}[1.2,8.1])$, and likely to have received less intraoperative opioids (intravenous morphine equi-analgesia: median $=5.8$ [0.1-8.2] vs. 
$11.7[3.7-19.0] \mathrm{mg}, p=0.03)$. Further, patients who did not survive admission were more like to have required an urgent medical review prior to RRT activation ( $62 \%$ vs. $40 \%, O R=2.4, p=0.05,95 \% \mathrm{Cl}[1.1$, 5.6]), compared to those who survived. There were no significant differences observed between those who survived and those who died in regard to the type of surgery or anesthesia (regional vs. general), number of perioperative hypotension episodes, or use of vasoactive medications, inotropes, or fluid therapy. Similarly, there were no observed statistical differences in the time from surgery to RRT activation or in unplanned admissions to critical care services (ICU or HDU).

\section{Discussion}

\section{Key findings}

We performed a retrospective observational study describing the perioperative characteristics of patients who required RRT activation after major hip surgery. We found that in-hospital mortality after RRT activation occurred in 1 in 7 patients. Moreover, we found that common patient characteristics associated with such activation included advanced age ( $>82$ years), frailty, high $\mathrm{CCl}$ score, and emergency surgery presentation. Finally, we found that overall mortality was close to 1 in 7 patients.

\section{Relationship to other studies}

To date, there have been no studies investigating RRT activation after major hip surgery. There is also limited research focusing on the perioperative determinants of postoperative RRT activation after major surgery. Three studies have identified perioperative characteristics affecting patient deterioration in the postoperative setting (5-7). Lee et al. conducted a retrospective case-control study investigating early postoperative emergencies requiring an intensive care team intervention (7), with 34 RRT activations identified for 32 patients. In the study, RRT participants were matched with a nested cohort of 126 controls. Similar to our findings, there were significant preoperative associations with early RRT activation, such as high ASA status. The authors did not report on frailty or detailed patient comorbidity. Likewise, the associations with perioperative hypotension, and detailed anesthesia and surgical variables, were not assessed.

Weingarten et al. performed a retrospective case-control study investigating patient characteristics and outcomes associated with RRT activation within the first 48 hours after surgical interventions (6). In the study, 181 patients were identified and matched to 318 controls. In contrast to our findings, which showed that approximately a quarter of our cohort had RRT activation within the first 12 hours postoperatively, Weingarten et al. reported that more than $60 \%$ of postoperative RRT activations occurred within the first 12 hours. The study cohort of Weingarten et al. also had a lower mean age (59 years vs. 82 years in our study), and their patient cohort had fewer comorbidities than our patient group. Through a multivariate analysis, Weingarten et al. showed preoperative opioid use, history of central neurologic disease, and intraoperative hemodynamic instability to be associated with postoperative decompensation requiring RRT activation. Of the 181 patients who had an RRT activation in their study, only 62 (34\%) underwent orthopedic surgery. The type of orthopedic surgery was also not specified. 
Differences in time to RRT activation may be explained by Weingarten et al. having only included RRT activations within the first 48 hours postoperatively and different patient demographics via the inclusion of all surgical procedures and not only major hip procedures.

More recently, in a tertiary children's hospital, Barry et al. performed a retrospective review of 100 RRT calls occurring within 24 hours of receiving anesthesia or procedural sedation (5). These patients' medical records were reviewed to obtain patient characteristics, etiology of the RRT call, and outcomes. Only nine patients (9\%) had undergone orthopedic surgery, the type of which was not specified. The authors reported that high ASA status, general anesthesia administration, and the presence of acute or chronic conditions prior to anesthetic administration predisposed a patient to perioperative complications resulting in the need for an RRT review. Generalization to patients undergoing major hip surgery is limited by the lack of detailed anesthesia and surgical variables reported. Further, the pediatric context and low prevalence of orthopedic patients in their study limits its generalization to our adult population.

\section{Study implications}

Our findings show that patients who required RRT activation after major hip surgery had an in-hospital mortality of $14 \%$. These patients were likely to be older, frail, have multiple comorbidities, and undergoing nonelective surgery. Intraoperative hypotension and the use of vasoactive medications was ubiquitous, and surgery was frequently performed after hours. Specifically, mortality after RRT activation following major hip surgery in our institution occurred in a significantly high-risk patient cohort, with a patient profile of advanced age (> 82 years), frailty, and high CCl being pervasive. Further, despite these important risks, such patients had no structured critical care support. The identification of such patients may allow for effective preoperative risk stratification, optimization of medical comorbidity, proactive planning regarding advanced care directives, and increased postoperative monitoring. Interestingly, we found that surgical factors (including type or duration of surgery) and anesthesia factors (including type of anesthesia, intraoperative hemodynamics, opioid use, and vasopressor use) did not differentiate patients who survived or died after RRT activation. These findings may be of particular interest to perioperative clinicians and health organizations, as they highlight-in patients for whom escalation of care is appropriate-opportunities for patient risk stratification and appropriate allocation of critical care resources (HDU or ICU) in the postoperative setting.

There has been a strong association reported between postoperative RRT activation and after-hours surgery (7); however, in our study, the majority of surgeries (77\%) were undertaken during normal working hours. Further, almost all patients who required RRT activation were discharged postoperatively to a general surgical ward, and over one third of our patient cohort required an urgent medical review prior to RRT activation. These findings may be of particular interest to perioperative clinicians and health organizations, as they highlight-in patients for whom escalation of care is appropriate-opportunities for patient risk stratification and appropriate allocation of critical care resources (HDU or ICU) in the postoperative setting. Our findings further highlight the need for enhanced postoperative ward surveillance and more effective early warning systems detecting postoperative patient deterioration. 
We reported on detailed perioperative hemodynamic variables: in particular, the use of invasive hemodynamic monitoring, rate of perioperative hypotension, and the use of fluid and vasoactive medications. Hypotension has been reported to be the most common indication for RRT activation in adult postsurgical patients $(6,7)$. There is mounting interest in intraoperative hypotension and its strong association with postoperative morbidity and mortality $(14,15)$. Over half of our patients experienced an episode of intraoperative hypotension, with $9 \%$ of patients having a severe hypotensive event. The almost ubiquitous use of invasive blood pressure monitoring possibly allowed for timely identification and treatment of perioperative hypotension, reflected in the frequent use of vasopressor medication, which was administered in nearly all patients (88\%). The use of a regional technique in $69 \%$ of patients may have also affected the frequency of vasoactive medication use. Postoperatively, in the PACU, almost half of the patients had a documented hypotensive event, and only $10 \%$ of patients required vasopressor support. Intraoperative vasopressor use has been associated with postoperative RRT requirement (6). We also identified that, compared to those who survived, patients who died received a lesser intravenous morphine equi-analgesia dose. This likely reflects the more advanced age and significantly higher comorbidity profile of these patients. However, analgesia use outside of the operating theater and PACU was not collected.

\section{Strengths and limitations}

There are several strengths and limitations of this study. This is a single-center study of patients undergoing major hip surgery, performed in a high-volume center for orthopedic surgery, which limits the external validity of our findings to other institutions and to other types of surgery. Importantly, given the exploratory design of this study, we only collected data on patients who had an RRT activation after major hip surgery. We did not compare these patients to those who underwent similar surgeries but did not have an RRT activation, which limits the broad application of our findings to all patients undergoing hip surgery. We did not collect data on comorbidity severity outside that of kidney disease and liver disease, which limits the detail of comorbidity description. The large number of distinct variables collected gives a detailed overview but severely limits the extrapolation of any causal relationships between perioperative course, adverse postoperative events, RRT activation, and post-RRT activation mortality.

However, our study also has several strengths. To date, this is the largest review of patients undergoing RRT activation after major hip surgery that specifically combines detailed patient, surgical, and anesthesia factors describing the perioperative course of patients. The collection of data on detailed preoperative comorbid conditions, including frailty, provides a comprehensive evaluation of the baseline health characteristics of our patient population. The detailed overview and rates of hypotension, vasopressor use, and use of opioids provide an in-depth insight into this patient cohort and their perioperative journey. By reporting the rate of RRT activation and mortality in these patients, we have defined both a need for enhanced postoperative ward surveillance and more effective early warning systems detecting postoperative patient deterioration. Our findings are hypothesis-generating and may provide valuable data for power calculations in future studies on RRT activation in the major orthopedic 
setting. Given the exploratory nature of the study, we cannot establish a causal relationship between the perioperative variables we assessed and their impact on RRT activations or any other postoperative outcomes. However, our in-depth insights, comparing patients who survived hospital admission to those who died, may provide data for sample size calculations for future RRT prospective studies.

\section{Conclusion}

Death following RRT activation occurred in 1 in 7 patients who had undergone major hip surgery in a tertiary referral hospital. We have identified several important findings relevant to RRT activation after major hip surgery. Most patients were older (> 82 years), frail, with a high $\mathrm{CCl}$, and undergoing out-ofhours emergency surgery. Our findings suggest that surgical (type or duration) and anesthesia (type, intraoperative hemodynamics, opioid use, vasopressor use) factors do not differentiate patients who died or survived following RRT activation. Given the high rate of RRT activations and high mortality rate after these activations, the findings, specific to major orthopedic surgery, provide good opportunities for the implementation of strategies aimed at improving postoperative outcomes in these at-risk patients.

\section{Abbreviations}

RRT: Rapid Response Team

CCl: Charlson Comorbidity Index

CSHA: Canadian Study of Health and Aging

PACU: Post Anesthesia Care Unit

SD: Standard Deviation

IQR: Interquartile Range

ASA: American Society of Anesthesiologists

ICU: Intensive Care Unit

HDU: High Dependency Unit

IV: Intravenous

\section{Declarations}

Ethics approval and consent to participate

Ethics approval was given by the Austin Health Human Research Ethics committee (LNR/17/Austin/616) prior to initiation of the study. The need for informed written consent from participants was waived due to 
the observational and retrospective nature of the study.

\section{Consent for publication}

Not applicable.

\section{Availability of data and material}

The datasets generated and analysed during the study are not publicly available due to individual privacy concerns but are available from the corresponding author on reasonable request.

\section{Competing interests}

The authors declare that they have no competing interests.

\section{Funding}

No external funding was sourced for this study.

\section{Authors' contributions}

LW, AP: Study and protocol design, trial governance, Human Research Ethics Submission, data analysis and interpretation, statistical analyses, writing of manuscript.

$\mathrm{RB}, \mathrm{AH}, \mathrm{IH}, \mathrm{COOT}, \mathrm{JN}, \mathrm{LE}, \mathrm{RH}$ : collection of perioperative data, data interpretation, preparation of manuscript.

LC: Study design, statistical analyses, data interpretation, writing of manuscript.

All authors have read and approved the manuscript.

Acknowledgements

Not applicable.

\section{References}

1) Devita MA, Bellomo R, Hillman $K$, Kellum J, Rotondi A, Teres $D$, et al. Findings of the first consensus conference on medical emergency teams. Crit Care Med. 2006; 34(9):2463-78.

2) Maharaj R, Raffaele I, Wendon J. Rapid response systems: a systematic review and meta-analysis. Crit Care. 2015; 19(1): 254

3) Robertson M, Chung W, Huynh A, O'Halloran T, Warrillow S, Gow P, et al. Medical emergency team activation in patients pre-and post-liver transplantation is associated with prolonged length of stay and 
higher morbidity. J Hepatol [internet]. 2017 [cited 2018 Mar 15];66(1):S189. Available from: https://linkinghub.elsevier.com/retrieve/pii/S0168827817306682

4) Desborough JP. The stress response to trauma and surgery. $\mathrm{Br} J$ Anaesth. 2000;85(1):109-17.

5) Barry N, Miller K, Ryshen G, Uffman J, Taghon TA, Tobias JD. Etiology of postanesthetic and postsedation events on the inpatient ward: data from a rapid response team at a tertiary care children's hospital. Paediatr Anaesth. 2016;26(5):504-11.

6) Weingarten TN, Venus SJ, Whalen FX, Lyne BJ, Tempel HA, Wilczewski SA, et al. Postoperative emergency response team activation at a large tertiary medical center. Mayo Clin Proc. 2012;87(1):41-9.

7) Lee A, Lum ME, O'Regan WJ, Hillman KM. Early postoperative emergencies requiring an intensive care team intervention. The role of ASA physical status and after-hours surgery. Anaesthesia. 1998;53(6):529-35.

8) NB Authority. Blood Management Guidelines: Module 2 Perioperative. 2012 [Available from: http://www.nba.gov.au/guidelines/module2/po-mod2.pdf2].

9) Moghissi ES, Korytkowski MT, DiNardo M, Einhorn D, Hellman R, Hirsch IB, et al. American Association of Clinical Endocrinologists and American Diabetes Association consensus statement on inpatient glycemic control. Endocr Pract. 2009;15(4):353-69.

10) Charlson ME, Pompei P, Ales KL, MacKenzie CR. A new method of classifying prognostic comorbidity in longitudinal studies: development and validation. J Chronic Dis. 1987;40(5):373-83.

11) Child CG, Turcotte JG. Surgery and portal hypertension. Major Probl Clin Surg. 1964;1:1-85.

12) Rockwood K, Song X, MacKnight $C$, Bergman H, Hogan DB, McDowell I, et al. A global clinical measure of fitness and frailty in elderly people. Cmaj. 2005;173(5):489-95.

13) von Elm E, Altman DG, Egger M, Pocock SJ, Gotzsche PC, Vandenbroucke JP, et al. The Strengthening the Reporting of Observational Studies in Epidemiology (STROBE) statement: guidelines for reporting observational studies. Lancet. 2007;370(9596):1453-7.

14) Monk TG, Bronsert MR, Henderson WG, Mangione MP, Sum-Ping ST, Bentt DR, et al. Association between Intraoperative Hypotension and Hypertension and 30-day Postoperative Mortality in Noncardiac Surgery. Anesthesiology. 2015;123(2):307-19.

15) Walsh M, Devereaux PJ, Garg AX, Kurz A, Turan A, Rodseth RN, et al. Relationship between intraoperative mean arterial pressure and clinical outcomes after noncardiac surgery: toward an empirical definition of hypotension. Anesthesiology. 2013;119(3):507-15.

\section{Figures}


Patients undergoing orthopedic surgery between Sept 2014-Nov 2017

$$
\text { ( } n=8094)
$$
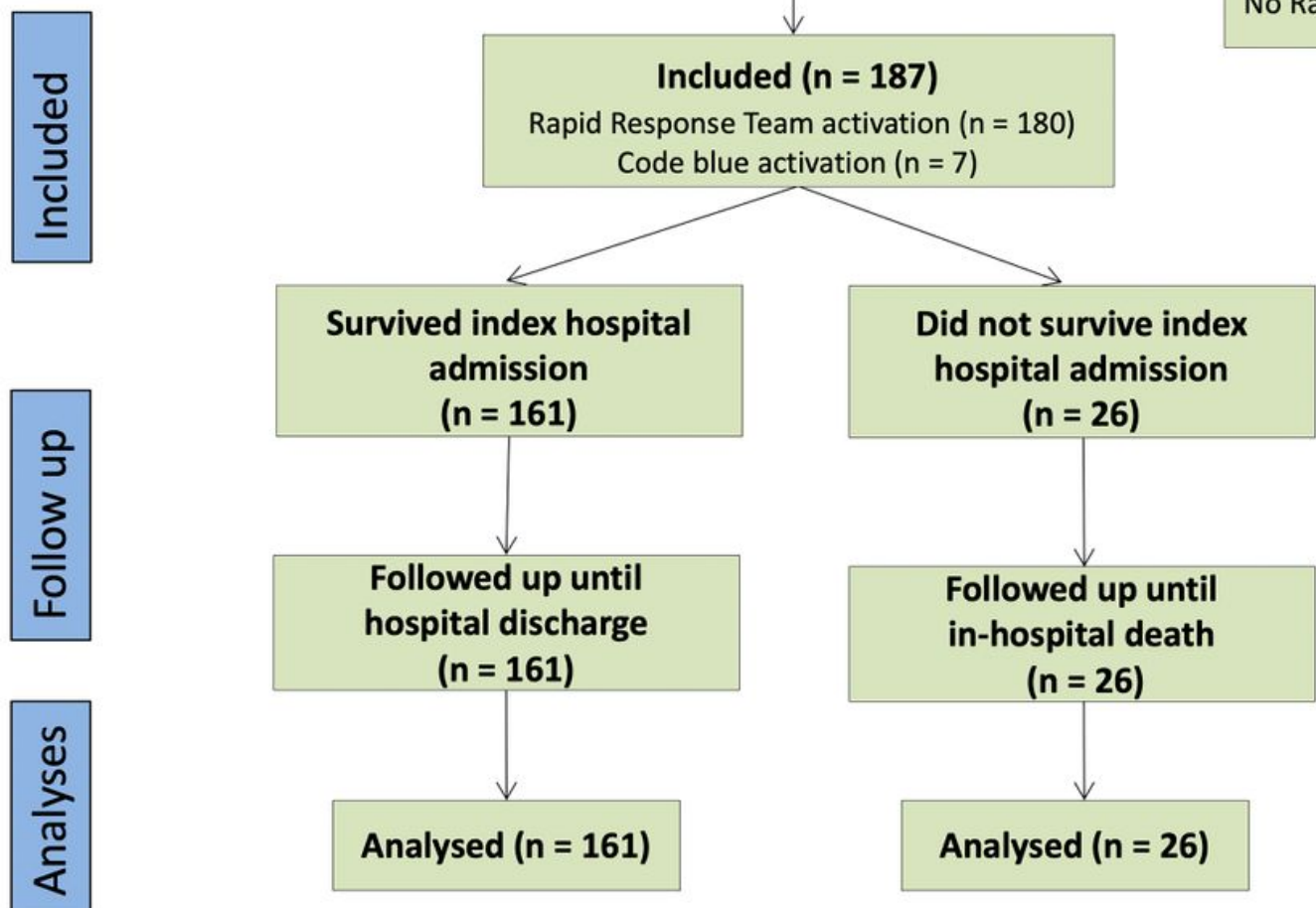

$$
\text { Included ( } \mathbf{n}=\mathbf{1 8 7})
$$

Rapid Response Team activation ( $n=180$ ) Code blue activation $(n=7)$

Excluded $(n=1638)$

Superficial/minor hip procedures $(n=39)$ No Rapid Response Team activation ( $n=1599$ )

Figure 1

Study flow diagram 